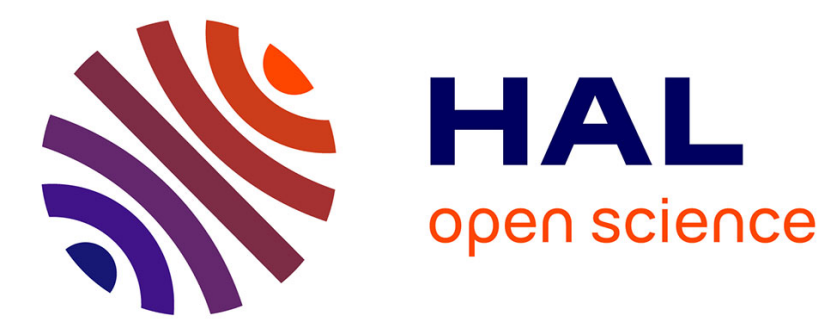

\title{
Propriétés magnétiques anisotropes du composé Tb0,37Y2,63Fe5O12
}

M. Lahoubi, G. Fillion

\section{To cite this version:}

M. Lahoubi, G. Fillion. Propriétés magnétiques anisotropes du composé Tb0,37Y2,63Fe5O12. Journal de Physique IV Proceedings, 1997, 07 (C1), pp.C1-287-C1-288. 10.1051/jp4:19971113 jpa-00254753

\section{HAL Id: jpa-00254753 https://hal.science/jpa-00254753}

Submitted on 1 Jan 1997

HAL is a multi-disciplinary open access archive for the deposit and dissemination of scientific research documents, whether they are published or not. The documents may come from teaching and research institutions in France or abroad, or from public or private research centers.
L'archive ouverte pluridisciplinaire HAL, est destinée au dépôt et à la diffusion de documents scientifiques de niveau recherche, publiés ou non, émanant des établissements d'enseignement et de recherche français ou étrangers, des laboratoires publics ou privés. 


\title{
Propriétés magnétiques anisotropes du composé $T b_{0,37} Y_{2,63} F_{5} O_{12}$
}

\author{
M. Lahoubi et G. Fillion*
}

Laboratoire de Magnétisme, Institut de Physique, Université de Annaba, BP. 12, El-Hadjar, 23000 Annaba, Algérie

* Laboratoire de Magnétisme Louis Néel, CNRS-UJF, 25 avenue des Martyrs, BP. 166, 38042 Grenoble cedex 9, France

\begin{abstract}
Résumé. Des mesures d'aimantation ont été effectuées sur le composé monocristallin $\mathrm{Tb}_{0.37} \mathrm{Y}_{2.63} \mathrm{Fe}_{5} \mathrm{O}_{12}$ dans l'intervalle de température $4.2 \mathrm{~K}-300 \mathrm{~K}$ en appliquant des champs magnétiques statiques selon les directions cristallographiques $\langle 111\rangle$ et $<100\rangle$. La comparaison des variations isothermes de l'aimantation en champs moyens ( 80 koe) a permis de montrer qu'en dessous de $120 \mathrm{~K},<100>$ est bien la direction facile spontanée. La forte anisotropie de l'aimantation qui apparait en dessous de $60 \mathrm{~K}$ semble liée à 1 'existence d'une structure non colinéaire spontanée des moments de terbium. Lorsqu'un champ magnétique intense (200koe) est appliqué selon la direction $<100>$, deux transitions magnétiques apparaissent à $4.2 \mathrm{~K}$ et disparaissent au delà de $16 \mathrm{~K}$. La première transition au champ critique $\mathrm{H}_{\mathrm{c}}=125 \mathrm{koe}$ qui présente les caractéristiques d'une transition du type "crossover" est analysée suivant le modèle théorique de Cooper. La deuxième transition au champ critique $\mathrm{H}_{02}=177 \mathrm{koe}$ s'explique par une réorientation de spin des sous réseaux fers en phase oblique. On montre enfin qu'un modele pour les composés Tb-YIG se rapproche plutôt d'un modèle XY que d'un modèle d'Ising.
\end{abstract}

\section{ASPECT EXPERIMENTAL}

Des mesures d'aimantation ont été effectuées sur une sphère monocristalline (diamètre $=3.1 \mathrm{~mm}$ ) de ferrite grenat de terbium substitué à l'Yttrium de formule $\mathrm{Tb}_{0.37} \mathrm{Y}_{2.63} \mathrm{Fe}_{5} \mathrm{O}_{12}$ de masse $41.3 \mathrm{mg}$, en utilisant deux appareillages différents par la gamme de champ mais qui utilisent la méthode d'extraction axiale de l'échantillon en pratiquant des variations thermiques entre $1.5 \mathrm{~K}$ et $300 \mathrm{~K}$. Le premier appareillage produit un champ magnétique continu maximum de 80koe qui est fourni par une bobine supraconductrice. Le second appareillage permet d'effectuer des mesures jusqu'à $200 \mathrm{koe}$ en champ continu au moyen d'une bobine résistive. Le champ magnétique est appliqué suivant les directions cristallographiques $<111>$ et $<100>$. Ces orientations s'obtiennent par la méthode de Laue avec une incertitude qui n'excède le degré. Les résultats sont présentés en fonction du champ magnétique interne $H$ que l'on obtient en retranchant au champ magnétique appliqué le champ démagnétisant calculé à partir du facteur de champ démagnétisant d'une sphère. L'aimantation $\mathrm{M}$ est défini en magnéton de Bohr pour deux formules $\mathrm{Tb}_{0.37} \mathrm{Y}_{2.63} \mathrm{Fe}_{5} \mathrm{O}_{12}$.

\section{RESULTATS ET DISCUSSION}

Les variations isothermes de l'aimantation selon les directions $\langle 111\rangle$ et $\langle 100\rangle$ en champs moyens (80koe) sont reportées sur la figure 1a. Selon la direction $\langle 111\rangle$, on observe des lois d'aimantation correspondant à des processus d'aimantation par rotation de l'aimantation à partir de la direction spontanée. Nous pouvons donc définir par extrapolation en champ interne nul d'une part l'aimantation $\mathrm{M}_{0}<111>$ pour les champs faibles et d'autre part l'aimantation à saturation technique $\mathrm{M}_{\mathrm{S}}<111>$ pour les champs élevés. On constate que ces aimantations se rejoignent à partir de $100 \mathrm{~K}$ et coïncident au delà de $120 \mathrm{~K}$. L'approche à saturation plus rapide selon $<100>$ montre que cette direction est bien la plus facile en dessous de $120 \mathrm{~K}$. Elle le reste jusqu'à très basse température comme l'atteste la comparaison des courbes à $1.5 \mathrm{~K}$. La valeur de $\mathrm{M}_{\mathrm{S}}<100>$ vaut $4.88 \pm 0.05 \mu_{\mathrm{B}} /$ mole à $1.5 \mathrm{~K}$, ce qui donne $5.12 \pm 0.05 \mu_{\mathrm{B}} /$ mole pour la résultante $\mathrm{M}_{R}$ des moments de terbium. Ceci correspond à une réduction moyenne de moment de $23 \%$ par rapport à l'ion libre. Selon toute vraisemblance, la structure magnétique spontanée des moments terres rares est donc non colinéaire à basse température. Au dessus de $60 \mathrm{~K}$, les courbes se rejoignent au delà de $10 \mathrm{koe}$ et coïncident en champs plus élevés. Elles mettent en évidence une forte anisotropie de l'aimantation $\Delta \mathrm{M}_{\mathrm{a}}=\mathrm{M}_{\mathrm{S}}<100>-\mathrm{M}_{\mathrm{S}}<111>$ qui devient non mesurable par cette méthode au delà de cette température. 

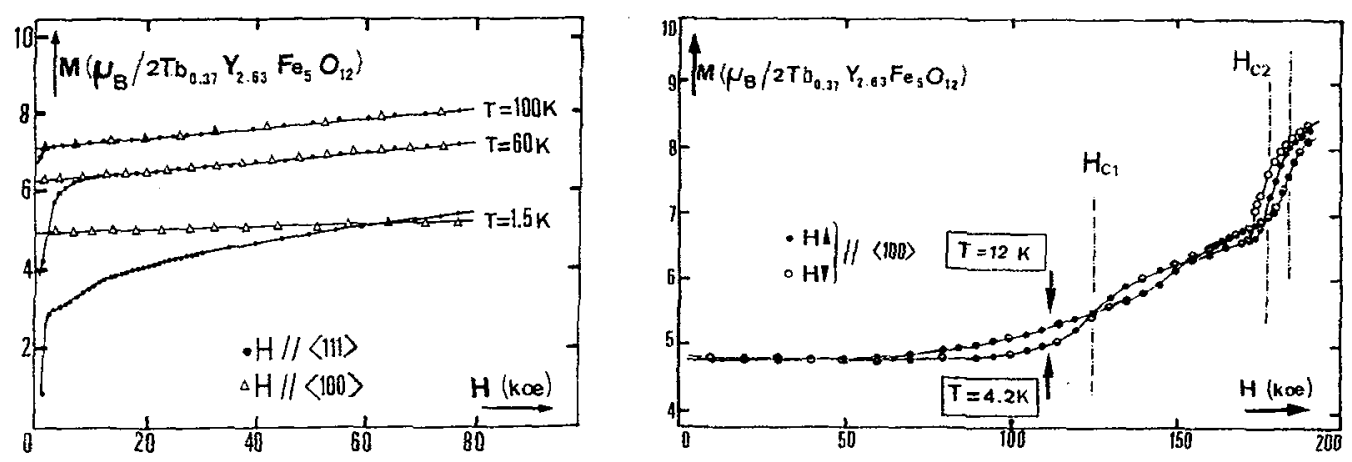

Figure 1: a-Mesures d'aimantation selon $<111>$ et $<100>$ en champs moyens ( 80 koe) à $1.5,60$ et $100 \mathrm{~K}$.

b- Mesures d'aimantation selon $<100>$ en champs intenses $(200$ koe) à 4.2 et $12 \mathrm{~K}$.

Les courbes tracées sur la figure $1 \mathrm{~b}$ mettent en évidence deux transitions magnétiques qui sont bien marquées à 4.2K avec des variations d'aimantation importantes, mais qui disparaissent à $16 \mathrm{~K}$. Cooper [1] a fait remarquer que pour certains composés de terres rares, les niveaux d'énergie des ions terre rare pouvaient se croiser sous l'effet du champ, entraînant une transition avec saut d'aimantation si la température est suffisamment basse. Une telle situation semble être réalisée dans les ferrites grenats substitués de $\mathrm{Tb}_{\mathrm{x}} \mathrm{Y}_{3-\mathrm{x}} \mathrm{IG}$ pour des concentrations $\mathrm{x}<0.5[2,3]$ et ce phénomène ("crossover") a été invoqué comme explication possible de certains sauts d'aimantation observés sur le composé $\mathrm{x}=0.26[4,5]$. Mais l'analyse faite par Nekvasil et al [6] ne permet pas de conclure définitivement en raison de la nature transitoire du champ pulsé utilisé dans les mesures. Il montre cependant qu'il faut tenir compte de l'anisotropie de l'échange si on veut rendre compte théoriquement de telles transitions. Dans notre cas, la première transition que nous observons en champ statique sur notre échantillon au champ critique $\mathrm{H}_{\mathrm{cl}}=125 \mathrm{koe}$ semble présenter toutes les caractéristiques d'une transition due à un tel "crossover" : i) il n'y a pas d'hystérésis observé qui serait contraire à la nature microscopique du phénomène ; ii) le point d'inflexion se produit au même champ qu'elle que soit la température; iii) l'évolution rapide des courbes avec la température correspond bien avec le modèle théorique simple de Cooper. La deuxième transition qui s'amorce pour un champ critique plus élevé $\left(\mathrm{H}_{\mathrm{c} 2}=177 \mathrm{koe}\right)$ présente une hystérèse de 5 koe à $4.2 \mathrm{~K}$ qui disparaît au delà de $12 \mathrm{~K}\left(\mathrm{H}_{\mathrm{c} 2}=183 \mathrm{koe}\right)$. Il est donc vraisemblable que se soit une réorientation du fer en phase oblique. En l'absence d'anisotropie, le champ critique serait en effet égale à $|\mathbf{n}| \mathrm{M}_{\mathrm{S}} \approx 100 \mathrm{koe}$ à $0 \mathrm{~K}$. Comme le champ est selon une direction facile et que l'énergie d'anisotropie est du même ordre de grandeur que l'échange, le champ de transition théorique peut être de 1.5 fois à 2 fois plus grand et la transition peut devenir du type métamagnétique avec un hystérésis prononcé, ce qui est bien le cas.

D'après les deux moments $\mathbf{m}_{1}$ et $\mathbf{m}_{1}^{\prime}$ trouvés pour "le double parapluie" selon $\langle 111\rangle$ dans TbIG [7], en peut en tenant compte d'un modèle quadratique [8], extrapoler une valeur assez approchée du moment $\mathbf{m}_{3}$ à savoir $\mathbf{m}_{3}=\mathbf{m}_{3}^{\prime}=8.1 \mu_{\mathrm{B}}$. Avec une réduction moyenne de moment qui correspond à un moment résultant de $6.93 \mu_{\mathrm{B}} / \mathrm{Tb}$, on a $\mathrm{m}_{1}=8.5 \mu_{\mathrm{B}}$ avec un angle de $41.8^{\circ}$ avec l'axe $<001>$ du champ. Notons que si l'angle est de $45^{\circ}$, c'est à dire $\mathbf{m}_{1}$ aligné exactement sur $<011>$, le moment $\mathrm{m}_{1}$ serait de $8.97 \mu_{\mathrm{B}}$, ce qui est encore tout à fait plausible car cela correspond à l'hypothèse $\mathrm{g}_{\mathrm{y}}=0$. Un modèle pour les substitués au terbium se rapproche donc plutôt d'un modèle XY que d'un modèle d'Ising comme les substitués à l'holmium [9].

\section{Remerciements}

Nous remercions M. Guillot (LCMI Grenoble) de nous avoir prêté l'échantillon élaboré par J.M. Desvignes (LMOS CNRS Bellevue) et J.C Picoche (LCMI Grenoble) de nous avoir donné accès aux champs intenses.

\section{Références}

[1] Cooper B. R., Phys. Lett 22 (1966) 244-245.

[2] Lagutin A. S. and Dmitriev A. V., Sov. Phys. Solid .State 30 (1988) 1705-1709.

[3] Guillot M., Marchand A., Desvignes J. M., Le Gall H., Merceron T. and Guillaud M., Z. Phys. B 74 (1989) $335-339$.

[4] Demidov V. G., Levitin R. Z. and Popov Yu. F., Sov. Phys. Solid .State 18 (1976) 347.

[5] Valiev U. V., Krinchik G. S., Levitin R. Z. and Mukimov K. M., JETP. Lett 29 (1979) 214-218.

[6] Nekvasil V., Roskovec V. and Zounová F., J. Appl. Phys 49 (1978) 1471-1473.

[7] Lahoubi M., Guillot M., Marchand A., Tchéou F. and Roudaut E., I.E.E.E Trans. Magn. Mag 20 (1984) 1518-1520.

[8] Lahoubi M., Structures Magnétiques non colinéaires et transitions de phases dans les grenats ferrimagnétiques de

Terbium et d'Yttrium (Thèse de Doctorat d'Etat, Université Joseph Fourier, 1986) pp 215-224.

[9] Zvezdin A. K., Mukhin A. A. and Popov A. I., Sov. Phys. JETP 45 (1977) 573-580 